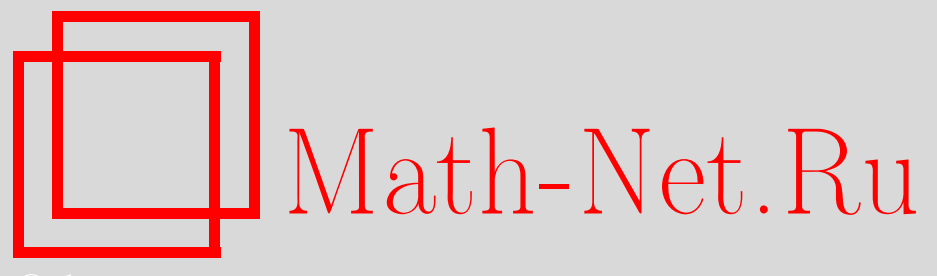

Г. В. Павлов, М. А. Кальмова, Е. С. Вронская, Эффект влияния продольных деформаций реологической балки на характер движения диска, Вестн. Сам. гос. техн. ун-та. Сер. Физ.-мат. науки, 2013, выпуск 1(), 253-259

DOI: https://doi.org/10.14498/vsgtu1158

Использование Общероссийского математического портала Math-Net.Ru подразумевает, что вы прочитали и согласны с пользовательским соглашением

http://www . mathnet.ru/rus/agreement

Параметры загрузки:

IP: 34.239 .49 .27

26 апреля 2023 г., 14:03:55

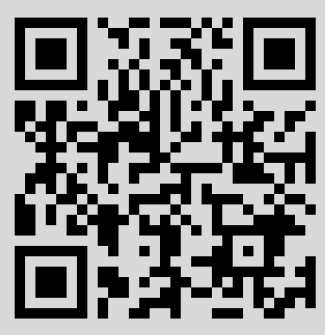


Вестн. Сам. гос. техн. ун-та. Сер. Физ.-мат. науки. 2013. № 1 (30). С. $253-259$

УДК 517.958+539.3(1)

\title{
ЭФФЕКТ ВЛИЯНИЯ ПРОДОЛЬНЫХ ДЕФОРМАЦИЙ РЕОЛОГИЧЕСКОЙ БАЛКИ НА ХАРАКТЕР ДВИЖЕНИЯ ДИСКА
}

\author{
Г. В. Павлов, М. А. Калъмова, Е. С. Вронская
}

Самарский государственный архитектурно-строительный университет, 443001, Россия, Самара, ул. Молодогвардейская, 194.

E-mails: senitskiy@mail.ru, kalmova@inbox.ru

Работа посвящена анализу влияния материала двухопорной реологической балки на динамику движущегося диска. Составлена гибридная система дифберенииальных уравнений, описывающих движение системы «диск - реологическая балка», состоящая из интегро-дифференииального уравнения продольных колебаний балки и уравнений в борме Лагранжа первого рода, которые определяют движение диска, а также уравнений неголономных связей, вытекающих из разности лагранжевых координат центра масс диска и точки балки, касающейся диска. Рассмотрен режим равномерного движения диска, что позволило проинтегрировать уравнение колебаний балки независимо от системы уравнений, описывающих движение диска. Показано, что при движении диска с малой скоростъю, а также в режиме, соответствующем предельному значению времени релаксации, в балке возникают физически неприемлемые дебормации. При нулевом времени релаксации наблюдается стационарный режим вынужденных колебаний балки при умеренных значениях амплитуд.

Ключевые слова: связъ неголономная, функиия Дирака, ядро релаксации, преобразование Лапласа.

Как известно $[1,2]$, при движении тела вращения по стержню, связанному с абсолютно жестким основанием, исключающим поперечные колебания, в стержне возникают продольные деформации, величина которых зависит от податливости материала стержня. В работе [1] была, в частности, поставлена задача о плоском движении диска по стержню на жестком основании с учетом продольных деформаций стержня. Представляет интерес оценка влияния продольных деформаций невесомого стержня длиной $L$, материал которого моделируется релаксационным телом Кельвина, на динамику диска (рис. 1).

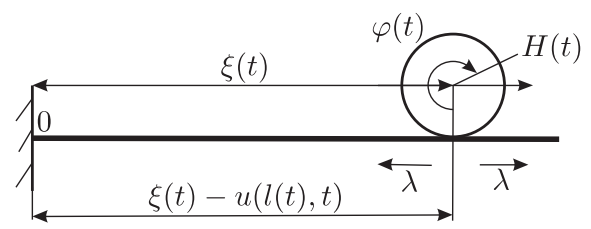

Рис. 1

Положение диска определим Лагранжевыми координатами $\xi(t)$ и $\phi(t)$, $u(x, t)$ - абсолютная деформация определенной части стержня. Лагранжеву координату точки касания диска обозначим через $l(t)$, определенную геомет-

Георгий Васильевич Павлов (к.ф.-м.н., доц.), доцент, каф. сопротивления материалов и строительной механики. Мария Александровна Кальмова, ассистент, каф. сопротивления материалов и строительной механики. Елена Сергеевна Вронская (к.ф.-м.н., доц.), доцент, каф. сопротивления материалов и строительной механики. 
рическим соотношением

$$
\xi(t)=l(t)+u(l(t), t)
$$

Из (1) и равенства $l(t)=R \dot{\varphi}$ вытекает соотношение

$$
R \dot{\varphi}=\dot{l}(t)\left[1+\frac{\partial u(x, t)}{\partial x}\right]_{x=l(t)}
$$

представляющее неголономную связь.

Для построения уравнения продольных колебаний реологического стержня запишем равенство, определяющее напряженно-деформированное состояние стержня в интегральной (релаксационной) форме [2].

Принимая во внимание известные равенства

$$
\begin{aligned}
& N=\sigma(x, t) \cdot F, \quad \frac{\partial N}{\partial x}=\rho F \frac{\partial^{2} u(x, t)}{\partial t^{2}}, \\
& \sigma(x, t)=E\left[\varepsilon_{x}(x, t)-\int_{0}^{t} \mathcal{R}(t-\tau) \varepsilon_{x}(x, \tau) d \tau\right] \\
& \mathcal{R}(t-\tau)=\frac{E-\bar{E}}{n E} \exp \left(-\frac{t-\tau}{n}\right),
\end{aligned}
$$

где $N, F$ и $\rho$-продольная сила, площадь поперечного сечения стержня и плотность материала стержня, $\sigma(x, t)$ - нормальное напряжение в поперечном сечении стержня на удалении $x$ от левого края стержня, $\varepsilon_{x}(x, t)$ - относительное удлинение стержня, $\mathcal{R}(t-\tau)$ - ядро релаксации материала, $n-$ время релаксации, $\bar{E}$ и $E$ - соответственно длительный и мгновенный модули упругости стержня на растяжение, построим уравнение движения реологического стержня постоянного сечения в проекции на ось $x$ :

$$
\frac{\partial^{2} u(x, t)}{\partial x^{2}}-\int_{0}^{t} \mathcal{R}(t-\tau) \frac{\partial^{2} u(x, t)}{\partial x^{2}} d \tau-\frac{1}{c^{2}} \frac{\partial^{2} u(x, t)}{\partial t^{2}}=\frac{1}{E F} \delta(x-l(t)) \cdot \lambda(t) .
$$

Здесь $\delta(x-l)$ - дельта-функция Дирака, $\lambda(t)$ - реологическая сила реакции в точке касания, $c^{2}=E / \rho$.

Уравнения движения диска представим в форме уравнений Лагранжа первого рода:

$$
m \ddot{\xi}(t)=H(t)-\lambda(t), \quad I \ddot{\varphi}(t)=\lambda(t) \cdot R,
$$

где $H(t)$ - активная движущая сил, $R$ - радиус диска, $I$ - аксиальный момент инерции диска. Без уравнений связи (1) и (2) постановка задачи будет неполной. Упрощая задачу, рассмотрим режим равномерного движения диска, принимая

$$
\xi=v t, \quad \ddot{\xi}=0, \quad \lambda=H=\text { const. }
$$

Здесь $v$-скорость центра масс диска. Равенство (4) имеет место при нулевых значениях главного вектора и главного момента системы сил, действующих на диск.

Принимая во внимание равенства (1), (3), (4), запишем уравнение 


$$
\begin{aligned}
\frac{\partial^{2} u(x, t)}{\partial x^{2}}-\int_{0}^{t} \mathcal{R}(t-\tau) \frac{\partial^{2} u(x, t)}{\partial x^{2}} d \tau-\frac{1}{c^{2}} \frac{\partial^{2} u(x, t)}{\partial t^{2}} & = \\
& =\frac{H}{E F} \delta\left(x-v t+\left.u(x, t)\right|_{x=v t}\right)
\end{aligned}
$$

Решение уравнения, описывающего свободные колебания реологического стержня

$$
\frac{\partial^{2} u_{0}(x, t)}{\partial x^{2}}-\int_{0}^{t} \mathcal{R}(t-\tau) \frac{\partial^{2} u_{0}(x, \tau)}{\partial x^{2}} d \tau-\frac{1}{c^{2}} \frac{\partial^{2} u_{0}(x, t)}{\partial t^{2}}=0
$$

при граничных

$$
\left.u_{0}(x, t)\right|_{x=0}=0,\left.\quad \frac{\partial u_{0}(x, t)}{\partial x}\right|_{x=L}=0
$$

и начальных условиях

$$
\left.u_{0}(x, t)\right|_{t=0}=f_{1}(x),\left.\quad \frac{\partial u_{0}(x, t)}{\partial t}\right|_{t=0}=f_{2}(x),
$$

разыскиваем в виде ряда

$$
u_{0}(x, t)=\sum_{m=1}^{\infty} X_{m}(x) T_{m}(t) .
$$

Подставляя (8) в уравнение (6) и разделяя переменные, приходим к уравнениям

$$
\begin{gathered}
\frac{d^{2} X_{m}(x)}{d x^{2}}+\lambda_{m}^{2} X_{m}(x)=0 \\
\frac{d^{2} T_{m}(t)}{d t^{2}}+p_{m}^{2} T_{m}(t)=p_{m}^{2} \int_{0}^{t} \mathcal{R}(t-\tau) T_{m}(\tau) d \tau \quad(m=0,1,2, \ldots)
\end{gathered}
$$

с краевыми

$$
\left.X_{m}(x)\right|_{x=0}=0,\left.\quad \frac{d X_{m}}{d x}\right|_{x=L}=0 \quad(m=0,1,2, \ldots)
$$

и начальными условиями

$$
\left.T_{m}(t)\right|_{t=0}=f_{1}(x),\left.\quad \frac{d T_{m}(t)}{d t}\right|_{t=0}=f_{2}(x) .
$$

Из (9) находим собственные числа

$$
\lambda_{m}=\frac{(2 m+1) \pi}{2 L} \quad(m=0,1,2, \ldots) .
$$

Решение уравнения (10) найдём, применяя преобразование Лапласа. В работе [2] при анализе свободных колебаний балки эта задача была решена, но, к сожалению, решение проведено при предельных значениях времени релаксации $(n=0, n=\infty)$, что практически исключает возможность учёта 
реологических свойств наследственного материала стержня, а именно в решение не вошли сомножители, придающие колебаниям затухающий характер. Обозначим оператор Лапласа $L\{T(t)\}=t(s)$. После применения Лапласовых преобразований к уравнению (10) запишем Лапласову трансформанту в виде

$$
t(s)=\frac{[s T(0)+\dot{T}(0)](n s+1)}{s^{3}+\frac{1}{n} s^{2}+p_{m}^{2} s-\frac{(E-\tilde{E}) p^{2}}{E n}}
$$

где $T(0)=f_{1}(0), \dot{T}(0)=f_{2}(0)$.

Найдём корни знаменателя: $s_{1}=\delta_{1}, s_{2,3}=-\delta_{2} \pm i p_{m}$. В этом случае оригинал $T_{m}(t)$ решения уравнения (8) имеет вид

$$
T_{m}(t)=B_{m} e^{-\delta_{1} t}+C_{m} e^{-\delta_{2} t}\left(\cos p_{m} t+\sin p_{m} t\right) .
$$

В результате решение начально-краевой задачи (6)-(7) представим в виде

$$
u_{0}(x, t)=\sum_{m=1}^{\infty}\left(b_{m} e^{-\delta_{1} t}+c_{m} e^{-\delta_{2} t}\left(\cos p_{m} t+\sin p_{m} t\right)\right) \sin \frac{(2 m+1) \pi}{2 L} x,
$$

где $b_{m}=A_{m} B_{m}, c_{m}=A_{m} C_{m}(m=0,1,2, \ldots)$. Значения коэффициентов $b_{m}$, $c_{m}$ определим из начальных условий $(7)$, используя свойство ортогональности собственных функций.

Исследуя вынужденные колебания, будем искать решение в виде ряда по собственным функциям однородной задачи:

$$
u_{\mathrm{H}}(x, t)=\sum_{k=0}^{\infty} \gamma_{k}(t) \sin \frac{(2 k+1) \pi}{2 L} x
$$

и подчиним его нулевым начальным и граничным условиям

$$
\left.u_{\mathrm{H}}(x, t)\right|_{t=0}=0,\left.\frac{\partial u_{\mathrm{H}}(x, t)}{\partial t}\right|_{t=0}=0,\left.u_{\mathrm{H}}(x, t)\right|_{x=0}=0,\left.\frac{\partial u_{\mathrm{H}}(x, t)}{\partial x}\right|_{x=L}=0 .
$$

После подстановки ряда (11) в уравнение (5) и разложения правой части уравнения в ряд по синусам по аргументу $x$ в интервале $(0, L)$ приходим к интегро-дифференциальному уравнению с нулевыми начальными условиями

$$
\frac{d^{2} \gamma_{k}(t)}{d t^{2}}-\omega_{k}^{2} \int_{0}^{t} \mathcal{R}(t-\tau) \gamma_{k}(\tau) d \tau+\omega_{k}^{2} \gamma_{k}(t)=q_{k}(t)
$$

где

$$
\begin{aligned}
q_{k}(t)=U \int_{0}^{L}\left[\delta(x-v t)+\gamma_{k}(t) \sin v_{k} t\right] & \sin \frac{(2 k+1) \pi x}{2 L}= \\
=U \eta\left(v t-\gamma_{k}(t) \sin v_{k} t\right) \eta & \left(L-v t+\gamma_{k}(t) \sin v_{k} t\right) \times \\
& \times \sin \left(\frac{(2 k+1) \pi\left(v t-\gamma_{k}(t) \sin v_{k} t\right)}{2 L}\right) .
\end{aligned}
$$


Здесь $U=\left(2 c^{2} H\right) /(E F L), v_{k}=[(2 k+1) \pi v] /(2 L), \omega_{k}=p_{k}=[(2 k+1) \pi c] /(2 L)$, $\eta$ - обобщённая функция Хевисайда. Удовлетворяя условиям функции Хевисайда и разлагая правую часть уравнения (12) в ряд Тейлора по малой ограниченной в окрестности нуля временной функции $\gamma_{k}(t)$, запишем уравнение (12) в виде

$$
\begin{aligned}
& \frac{d^{2} \gamma_{k}(t)}{d t^{2}}-\omega_{k}^{2} \int_{0}^{t} \mathcal{R}(t-\tau) \gamma_{k}(\tau) d \tau+\omega_{k}^{2} \gamma_{k}(t)= \\
& \quad=U\left[\sin \frac{(2 k+1) \pi v}{2 L}-\pi \frac{(2 k+1)}{4 L} \gamma_{k}(t) \sin \frac{(2 k+1) \pi v t}{L}\right] .
\end{aligned}
$$

Изображающее уравнение, соответствующее дифференциальной задаче (13) с правой частью при нулевых начальных условиях

$$
\begin{aligned}
\gamma_{k}(s)\left(s^{3}+\right. & \left.\frac{s^{2}}{n}+\omega_{k} s+\frac{\omega_{k}^{2}}{n} \frac{\tilde{E}}{E}\right)-\frac{(2 k+1) U \pi v}{4 n} \times \\
& \times\left(\frac{8 L}{4 L^{2} s^{2}+(2 k+1)^{2} \pi^{2} v^{2}}-\frac{\pi(2 k+1) \gamma_{k}(s)}{L^{2} s^{2}+(2 k+1)^{2} \pi^{2} v^{2}}\right)(1+n s)=0
\end{aligned}
$$

имеет частное решение - Лапласову трансформанту - вида

$$
\begin{aligned}
\gamma_{k}(s)=(2(1+ & 2 k) L \pi(1+n s) U v) \cdot\left(n\left(4 L^{2} s^{2}+(1+2 k)^{2} \pi^{2} v^{2}\right)\right) \times \\
& \times\left(s^{3}+\frac{(1+2 k)^{2} \pi^{2}(1+n s) U v}{4 n\left(L^{2} s^{2}+(1+2 k)^{2} \pi^{2} v^{2}\right)}+\frac{\tilde{E} \omega^{2}}{E n}+\frac{s^{2}}{n+\omega s}\right)^{-1} .
\end{aligned}
$$

Оригиналы функции $\gamma_{k}(s)$ найдены для двух частных случаев.

1. Скорость центра масс диска мала и трансформанту Лапласа можно представить равенством

$$
\gamma_{k}(s)=(1+2 k) \pi(1+n s) U v\left(2 \operatorname{Lns} s^{2}\left(s^{3}+\frac{\tilde{E} \omega^{2}}{E n}+\frac{s^{2}}{n+\omega s}\right)\right)^{-1}
$$

знаменатель которого имеет два кратных нулевых корня, а оригинал имеет вид

$$
\gamma_{k}(t)=d_{1} t+d_{2}+\sum_{k=3}^{6} d_{k} e^{s_{k} t}
$$

Значения $d_{k}$ и $s_{k}$ в общем виде не выписаны ввиду их громоздкости. Проведём численный анализ при следующих данных: $n=50 \mathrm{c}, E=1 \mathrm{H} / \mathrm{cm}^{2}, \tilde{E}=$ $=0,7 \cdot E \mathrm{H} / \mathrm{cm}^{2}, L=100 \mathrm{~cm}, \rho=0,05 \mathrm{\Gamma} / \mathrm{cm}^{3}, v=100 \mathrm{~cm} / \mathrm{c}, R=10 \mathrm{cM}$, $c=\sqrt{E / \rho} \mathrm{cm} / \mathrm{c}, \omega_{1}=(3 \pi c) /(2 L) \mathrm{c}^{-1}, p_{1}=\pi /(2 L) \sqrt{E / \rho} \mathrm{c}^{-1}, H(t)=2 H$, $F=1 \mathrm{~cm}^{2}, \delta_{1}=0,014 \mathrm{c}^{-1}, \delta_{2}=0,003 \mathrm{c}^{-1}, b_{1}=809 \mathrm{~cm}, c_{1}=-803 \mathrm{~cm}, k=1$, $x=10$ cм.

График изменения деформаций балки $u(x, t)$ для этого случая представлен на рис. 2. 


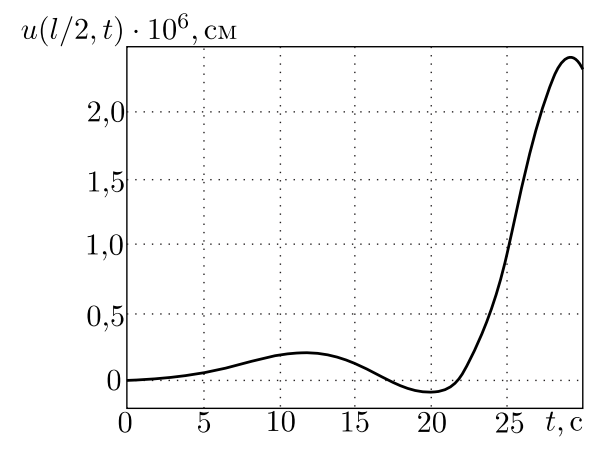

Рис. 2

Видим, что наличие физически неприемлемых амплитуд балки указывает, что режим плоского движения диска по реологической балке модели Кельвина не может быть реализован. Подобную картину можно наблюдать и при движении диска с малой скоростью, когда $n=\infty$.

2. При нулевом значении времени релаксации трансформанта (14) упрощается:

$$
\begin{aligned}
\gamma_{k}(s)=2 & (1+2 k) L \pi U v \times \\
& \times\left(\left(4 L^{2} s^{2}+(1+2 k)^{2} \pi^{2} v^{2}\right)\left(\frac{\pi^{2}(1+2 k)^{2} U v}{4 L^{2} s^{2}+(1+2 k)^{2} \pi^{2} v^{2}}+\frac{\tilde{E} \omega^{2}}{E}\right)\right)^{-1}
\end{aligned}
$$

Оригинал временной функции выражается через тригонометрические функции, не содержащие секулярных множителей.

В этом случае движение диска протекает в стационарном режиме вынужденных колебаний балки при умеренных значениях амплитуд.

Достоверность полученных результатов - наличие физически неприемлемых деформаций подтверждается совпадением их с результатами численного интегрирования интегро-дифференциальных уравнений (10), (12) как с ядром релаксации стандартного наследственного тела, так и с ядром слабосингулярной модели, предложенным А. Р. Ржаницыным [3].

\section{БИБЛИОГРАФИЧЕСКИЙ СПИСОК}

1. О. А. Горошко, "Неголономные системы с телами, что деформируются" // Вестн. Киев. yн-ma, 1983. № 25. C. 51-55. [O. A. Goroshko, "Nonholonomic systems with bodies that are deformed" // Vestn. Kiyev. Un-ta, 1983. no. 25. Pp. 51-55].

2. O. A. Goroško, K. Hedrih (Stevanović), Analitička dinamika (mehanika) diskretnih naslednih sistema (in Serbian). Niš: University of Niš, 2001. 426 pp.

3. А. Р. Ржаницын, Некоторые вопросы механики систем деформирующихся во времени. M.: Гостехиздат, 1949. 248 с. [A. R. Rzhanitsyn, Some Problems in the Mechanics of TimeDeformable Systems. Moscow: Gostekhizdat, 1949. 248 pp.]

4. R. M. Dreizler, C. S. Lüdde, Theoretical Mechanics: Theoretical Physics $1 /$ Graduate Texts in Physics. Berlin: Springer, 2011. 402 pp.

Поступила в редакцию $12 / \mathrm{XI} / 2012$;

в окончательном варианте - 25/I/2013. 
MSC: 70F25; 70E50

\title{
EFFECT OF THE INFLUENCE OF RHEOLOGICAL BEAM LONGITUDINAL STRAINS ON THE DISC MOTION STATE
}

\author{
G. V. Pavlov, M. A. Kalmova, E.S. Vronskaya
}

Samara State University of Architecture and Civil Engineering, 194, Molodogvardeyskaya st., Samara, 443001, Russia.

E-mails: senitskiy@mail.ru, kalmova@inbox.ru

The paper analyzes the effect that the material of a simple rheological beam has on the dynamics of a moving disc. The hybrid system of the differential equations describing the motion of the system disc-rheological beam consisting of the integro-differential equation of beam longitudinal vibrations and the Lagrange equations of the first kind, defining the motion of the disc, and the equations of nonholonomic constraints following from the difference between the Lagrange coordinates of the disc mass center and the beam point contacting with the disc is composed. The paper considers the mode of the disc steady motion, allowing to integrate the equation of beam vibrations regardless the system of equations describing the motion of the disc. It is identified that when the disc moves at a low speed, and in the mode corresponding to the limit value of the relaxation time it causes physically inadequate strain in the beam. When relaxation time is null there is a steady mode of forced beam vibrations at moderate amplitudes.

Key words: nonholonomic connection, Dirac delta function, relaxation kernel, Laplace transformation.

Original article submitted $12 / \mathrm{XI} / 2012$; revision submitted $25 / \mathrm{I} / 2013$.

Georgiy V. Pavlov (Ph. D. (Phys. \& Math.)), Associate Professor, Dept. of Strength of Materials and Structural Mechanics. Mariya A. Kalmova, Assistant, Dept. of Strength of Materials and Structural Mechanics. Elena S. Vronskaya (Ph.D. (Techn.)), Associate Professor, Dept. of Strength of Materials and Structural Mechanics. 